\title{
Agar pre-embedding of small skin biopsies: real-life benefits and challenges in high throughput pathology laboratories
}

\author{
Mara Ridolfi, ${ }^{1}$ Michele Paudice, ${ }^{1}$ Sandra Salvi, ${ }^{2}$ Luca Valle, ${ }^{3}$ Marina Gualco, ${ }^{2}$ \\ Antonio Perasole, ${ }^{4}$ Luca Anselmi, ${ }^{5}$ Roberto Fiocca, ${ }^{3,6}$ Luca Mastracci, ${ }^{3}$ Federica Grillo ${ }^{3}$
}

${ }^{1}$ Department of Surgical Sciences and Integrated Diagnostics (DISC), Univeristy of Genoa, Genoa, Italy

${ }^{2}$ Anatomic Pathology, Ospedale Policlinico San Martino IRCCS,

Genoa, Italy

${ }^{3}$ Anatomic Pathology,

Department of Surgical Sciences and Integrated Diagnostics

(DISC), Univeristy of Genoa, Genoa, Italy

${ }^{4}$ Anatomic and Cytopathology, Az. ULSS 8 Berica, Regione Veneto, Ospedale San Bortolo, Vicenza, Italy

${ }^{5}$ Anatomic Pathology, ASL 3, Ospedale Villa Scassi, Genoa, Italy

${ }^{6}$ Ospedale Policlinico San Martino IRCCS, Genoa, Italy

\section{Correspondence to}

Dr Federica Grillo, Anatomic Pathology, Department of Surgical Sciences and Integrated Diagnostics (DISC), University of Genoa and Ospedale Policlinico San Martino IRCCS, Genoa 16132, Italy; federica.grillo@ unige.it

MR, MP, LM and FG contributed equally.

Received 19 December 2018 Revised 22 January 2019 Accepted 23 January 2019 Published Online First 20 February 2019

\section{Check for updates}

(C) Author(s) (or their employer(s)) 2019. № commercial re-use. See rights and permissions. Published by BMJ.

To cite: Ridolfi M, Paudice M Salvi S, et al. J Clin Pathol 2019:72:448-451.

\begin{abstract}
Paraffin embedding of small, thin tissue samples requires specific expertise for optimal orientation before tissue sectioning. This study evaluates the real-life utility of the agar pre-embedding technique for small skin biopsies with regards to lengthening of work times, problems in orientation (re-embedding) and ancillary techniques (immunohistochemistry and in situ hybridisation) between two high work flow pathology laboratories, one of which routinely uses the agar pre-embedding technique and one which does not. The mean time required for pre-embedding in agar was $30.4 \mathrm{~s}$, but time for paraffin embedding for agar pre-embedded samples was shorter than the traditional method (177 vs $296 s ; p<0.005$ ). The number of skin samples requiring re-embedding was significantly higher with the traditional embedding method $(p<0.005)$. No problems in immunoreactivity were observed in all 1900 reactions performed with 17 different antibodies. Fluorescence in situ hybridisation analysis was optimised with a prolonged protease $\mathrm{K}$ incubation time (21 vs $18 \mathrm{~min}$ ).
\end{abstract}

\section{INTRODUCTION}

Automation in histopathology laboratories has greatly changed our day to day working lives enabling a quicker and more standardised work flow. However, in histopathology in particular, some steps within the entire process are still completely dependent on the manual performance, accuracy and capability of biomedical laboratory staff. In particular, paraffin embedding of tissue samples, especially small and thin fragments, requires specific expertise for optimal orientation before tissue sectioning. Errors in this phase may be addressed by melting the paraffin block and re-embedding tissue, but this can result in irreversible tissue loss.

For particularly small tissues or those which require precise orientation, pre-embedding techniques have been proposed. One of the most used mediums is agar, ${ }^{1-4}$ and this has been used for the embedding of various tissue samples. ${ }^{5}$

Agar is a neutral polysaccharide complex often used in bacteriology, extracted from the algae red agar Rhodophyceae, and is composed of about $70 \%$ agarose $/ 30 \%$ agaropectin. The point of pre-embedding is that the tissue is optimally oriented by the pathologist at the time of cut up, as tissues can be arranged or oriented as desired, and the sample is then fixed in position by covering with liquid agar, which then solidifies at room temperature. The pre-embedded tissue can subsequently be routinely processed and paraffin embedded as an agar-encased tissue.

This technique is by no means new, indeed the first reports are from the 1960s, however, despite objective usefulness it is little used in pathology. Perceived increase in cut up times and difficulty in managing agar are probably at the base of this as well as the possibility that agar may interfere with modern ancillary techniques.

In this study, we evaluated the real-life utility of the agar pre-embedding technique by comparing small skin biopsies in two high work flow pathology laboratories. Furthermore, evaluation was also extended to verify whether agar pre-embedding leads to problems in immunohistochemistry or fluorescence in situ hybridisation (FISH).

\section{MATERIALS AND METHODS Agar preparation and agar pre-embedding technique}

Agar is used as a 1\% solution of agarose (Roche, Basel, Switzerland) in distilled water. The solution is heated till boiling point and the resulting transparent fluid cooled for 24 hours. The agar, after its solidification, is divided into cubes and stored at $4^{\circ} \mathrm{C}$. When necessary, cubes are melted and the agar solution is kept liquefied at $60^{\circ} \mathrm{C}$ on a heating plate, ready for use. ${ }^{5}$ During cut up of small biopsies, specimens are placed and oriented on a refrigerated plate, and covered with liquid agar using a pipette. After a few seconds, the agar solidifies and the agar-embedded specimens are moved to the tissue cassette, preserving correct orientation. After routine processing, the agar pre-embedded tissue is finally embedded in paraffin and subsequently microtome cut (figure 1).

\section{Comparison of agar pre-embedding technique and traditional paraffin embedding of small skin biopsies}

To verify the advantage of using the agar pre-embedding technique, comparison was carried out between two high work flow pathology laboratories, one which routinely uses the agar-paraffin embedding technique (ASL3-Anatomic Pathology, ASL3, Genoa, Italy) for small skin biopsies while the other laboratory does not 


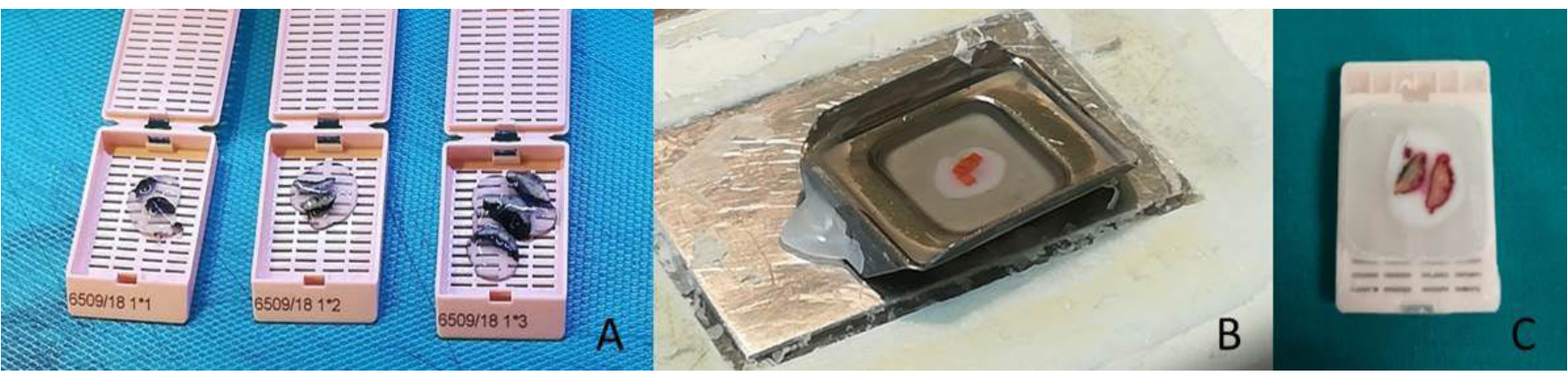

Figure 1 Agar pre-embedding technique. (A) Agar pre-embedded skin tissue samples in the plastic biocassettes. (B) Paraffin embedding of agar preembedded skin sample. (C) Paraffin block with agar pre-embedded skin sample; the white halo surrounding the skin sample is the agar medium.

(UNIV-Anatomic Pathology, University of Genoa, Genoa, Italy).

The following data from both laboratories were collected:

- Annual number of small skin biopsies (ASL3 and UNIV) and in the 4-month study period (June, July, September and October 2017).

- Number of small skin biopsies requiring re-embedding for tissue mal-orientation in the 4 months listed above (ASL3 and UNIV). Re-embedding is used as a quantifiable surrogate for severe problems in orientation.

- Time (mean in seconds) required for agar pre-embedding at the grossing station by the pathologist for small skin biopsies (evaluation carried out over a period of 1 week).

- Time (mean in seconds) required for paraffin embedding by an experienced technician for agar pre-embedded samples compared with non-agar embedded samples (evaluation carried out over a period of 1 week).

Differences in numbers of skin samples requiring paraffin re-embedding were compared between agar pre-embedded and traditional method using the $\mathrm{X}^{2}$ test while differences between times at the embedding station were tested using the student t-test $(\mathrm{p}<0.05$ for significance).

\section{Immunohistochemistry}

Data on all agar pre-embedded skin biopsies which required immunohistochemical evaluation were collected from the ASL 3 laboratory database in 4 months of the study period (June, July, September and October 2017). All immunoreactions were carried out using the routine, automated immunostainer BenchMark XT immunostainer (Ventana Medical Systems, Tucson, Arizona, USA) which permits standardised heat and enzymatic pretreatment as per protocol; ultraView Universal Alkaline Phosphatase Red Detection Kit was used (Ventana Medical Systems). Haematoxylin was used for counterstaining.

The following data were collected:

- Total number of immunoreactions performed on agar pre-embedded small skin biopsies in the 4-month period and types of antibodies used.

- Number of immunoreactions with problems sufficient to cause difficulties in evaluation such as inadequate immunoreaction, reduction of signal intensity, non-specific immune-signalling. A scoring system from -/- (negative immunosignal) to $+/+$ (strongly positive was used). ${ }^{6}$

- Which antibodies showed insufficient results and any differences in site of antigen expression (cytoplasmic, membranous, nuclear), antigen retrieval methods (heat or enzymatic pretreatment) or antibody characteristics.

\section{Fluorescence in situ hybridisation}

Little is known whether agar-encased tissue interferes with in situ hybridisation. Eight samples of melanoma on small skin biopsies which had been agar pre-embedded were selected. The Vysis (Abbott Molecular, Illinois, USA) Melanoma FISH Probe Kit (Vysis LSI PREB1/MYB/LSI CCND1/CEP6) is designed to detect copy numbers of the RRB1 (chromosome 6p25), MYB (chromosome 6q23), CCND1(chromosome 11q13) genes and of centromere 6 via FISH on formalin-fixed, paraffin-embedded tissue specimens as an aid in the diagnosis of melanoma.

The in situ hybridisation technique is briefly described below. From the selected agar pre-embedded paraffin-blocks, 5-micron thick sections were cut on polarised slides and deparaffinised using Abbott VP2000 (Abbott Molecular) processor which performs standardised preprogrammed protocols for deparaffinisation, pretreatment and staining. Considering that agar pre-embedding may lead to problems in digestion of the nuclear membrane to make DNA sequences available for hybridisation, two different protocols were compared. Changes were based on the length of incubation time for the protease $\mathrm{K}$ digestion step as detailed: (1) $18 \mathrm{~min}$ and (2) $21 \mathrm{~min}$. In situ hybridisation was carried out as per protocol and hybridisation by fluorescent signal was evaluated using the fluorescent microscope Olympus BX 61 (Olympus, Tokyo, Japan) with the Z-stack image acquisition system (Zeiss, Oberkochen, Germany). Only slide adequacy was evaluated using the following criteria: (a) probe signal intensity; (b) probe signal specificity and (c) background.

\section{RESULTS}

\section{Comparison of agar pre-embedding technique versus traditional paraffin embedding}

A synopsis of the skin sample numbers from both laboratories is provided in table 1 . The number of skin samples requiring re-embedding due to problems in tissue orientation was

Table 1 Table showing the number of skin samples sent to the ASL3 pathology laboratory (ASL3) and to the University of Genoa (UNIV) pathology laboratory, both annually and within the 4-month study period (mean and range)

\begin{tabular}{llc}
\hline & \multicolumn{2}{c}{ Number of skin samples } \\
\cline { 2 - 3 } & ASL 3 & UNIV \\
\hline Per year & 3235 & 5249 \\
4-month study period & 1053 & 1842 \\
Mean per month & 263 & 460 \\
Range & $182-347$ & $399-518$ \\
\hline
\end{tabular}




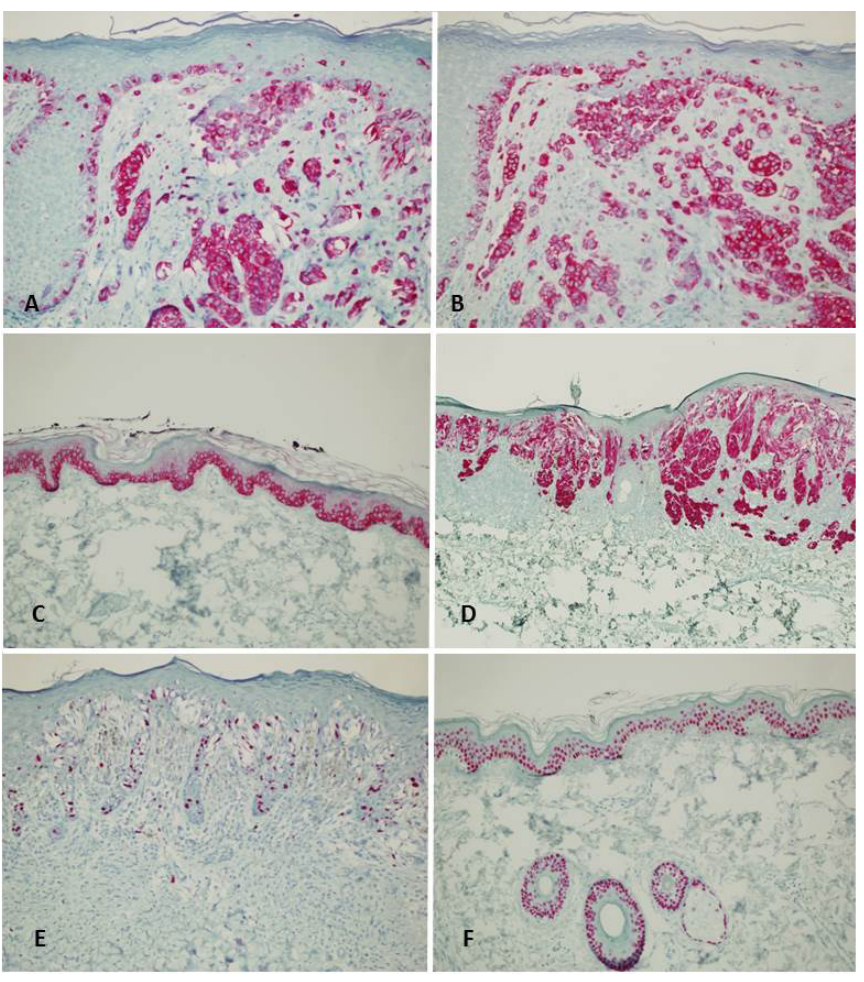

Figure 2 Immunohistochemistry on agar pre-embedded small skin biopsies (magnification X20); ultraView universal alkaline phosphatase red detection kit (Ventana Medical Systems). (A) Melan a positivity in an invasive malignant melanoma. (B) HMB 45 positivity in an invasive malignant melanoma. (C) Cytokeratin 5/6 cytoplasmic positivity in normal skin. (D) p16 nuclear and cytoplasmic positivity in a Spitz nevus. (E) Ki67 nuclear proliferation marker in a superficial spreading melanoma and (F) p63 nuclear positivity in normal skin.

significantly different $(\mathrm{p}<0.00001)$ between the agar pre-embedding technique $(1.42 \%-15 / 1053)$ and the traditional paraffin-embedding method (11.4\%-210/1842).

Regarding work times, mean time required for pre-embedding in agar at the time of cut up was 30.4 s, carried out by pathologists with more than 10-year experience who have been using agar pre-embedding for at least 1 year. At the paraffin-embedding station, after processing, the mean time for paraffin embedding for agar pre-embedded samples was significantly shorter if compared with the traditional method (agar pre-embedded tissue-177 s vs traditional paraffin embedding-296 s; p<0.005).

\section{Immunohistochemistry}

The total number of agar pre-embedded skin samples which were submitted for immunohistochemistry in the four study months was 463 samples (mean of 116/month) with a total of 1900 immunoreactions (mean of 475 sections/month).

The following antibodies were requested, showing a wide range of differential diagnoses including melanocyte lesion markers (HMB-45, MELAN-A, S-100 protein, p16 protein), lymphoid (CD20, CD3, CD30, CD4, CD8, light chains lambda and kappa), epithelial (wide spectrum cytokeratinsMNF116, low weight cytokeratins 34ßE12) and mesenchymal markers (vimentin, smooth muscle actin, CD34) and Ki67 for proliferation.

At re-evaluation of quality of immunoreactions, no problems sufficient to impair diagnoses were observed (figure 2A-F);
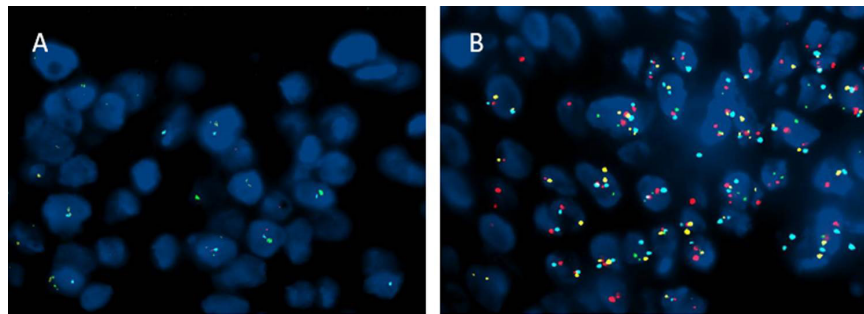

Figure 3 Fluorescent in situ hybridisation using the Vysis Melanoma FISH Probe Kit (Abbott Molecular, Illinois, USA). The RRB1 (6p25) probe is labelled with Spectrum Red; the MYB (6q23) probe is labelled with Spectrum Gold; the CCND1 (11q13) probe is labelled Spectrum Green; the CEP 6 probe is labelled Spectrum Aqua. (A) Protease K incubation for 18 min showing faint probe signal intensity (but appropriate specificity and no background). (B) Protease K incubation for $21 \mathrm{~min}$ showing more defined and evaluable signal with satisfactory probe signal intensity, correct specificity and no background.

antibodies for the light chains kappa and lambda showed background non-specific immunostaining, however, comparison with cases with no agar pre-embedding also showed similar background staining. No differences between various antibodies, sites of antibody reaction, antigen retrieval methods were observed, and overall, the method was comparable to immunoreactions performed on non-agar pre-embedded tissue.

\section{Fluorescence in situ hybridisation}

Samples were processed using the two protease incubation protocols described and compared. FISH performed with only 18 min of protease $\mathrm{K}$ time showed a faint probe signal intensity (but appropriate specificity and no background), which was difficult or impossible to interpret correctly (figure 3A). On the other hand, when protease $\mathrm{K}$ incubation time was increased to $21 \mathrm{~min}$, this resulted in a more defined and evaluable signal with satisfactory probe signal intensity, correct specificity and no background (figure $3 \mathrm{~B}$ ).

\section{CONCLUSIONS}

Optimal orientation of tissue within the paraffin block is mandatory for accurate histological diagnosis. Indeed, a frequent problem in routine pathology laboratories is the incorrect embedding of tissues in paraffin and this is especially true for small skin biopsies. The older literature from the 1960s, 1970s and 1980s proposed a pre-embedding technique in agar to maintain the precise orientation of tissue specimens before paraffin embedding. ${ }^{1-4}$ Advantages in microtome section preparation have been well documented in small tissue biopsies, ${ }^{7-9}$ ocular biopsies, ${ }^{10}$ skin samples ${ }^{11} 12$ and large dissection specimens. ${ }^{13}$ Unfortunately, even though often used in cytology ${ }^{14}$ and biology/botany, ${ }^{15}$ this technique is by no means widespread in routine pathology laboratories as it requires instrumentation, all be it simple, and lengthens cut up time.

The aim of the current study was to investigate the benefits and possible challenges of the agar pre-embedding technique when applied to skin samples. Its main purpose was to verify whether agar pre-embedding permits optimal orientation of small skin samples and to identify whether this technique is truly as time consuming as suggested. To be able to objectively quantify potential pitfalls in agar pre-embedding, we 
compared two high workload pathology laboratories used to dealing with numerous specimens daily.

Our data confirms that agar pre-embedding is advantageous in terms of orientation, with fewer samples requiring re-embedding due to mal-orientation. What is new is the quantification of time necessary for all the stages involved in the agar pre-embedding process from the grossing station to paraffin inclusion. While the perception that agar pre-embedding lengthens cut up times slightly (approximately $30 \mathrm{~s} / \mathrm{sample}$ ) is true, time saved at the paraffin-embedding station is about four times greater (approximately 119 s). A possible explanation for the fact that this time saving in the laboratory has up till now been overlooked is that no publication has meticulously evaluated the times required for each step in the pathology process. Correct tissue orientation coupled with time saving at paraffin embedding makes this technique advantageous in high workload pathology laboratories.

An added aspect, which confirms other reports in the literature $^{916}$ is that agar pre-embedding does not affect immunohistochemistry; indeed all reactions proved to be easily assessable and reliable. Furthermore, Yadav et al, report that coloured inks can be safely used with no undue spread within the tissue. ${ }^{17}$ In situ hybridisation, with easy tweaking of the procedure (slight lengthening of protease $\mathrm{K}$ incubation), also proved to be a safe and reliable method. Reports, admittedly from the older literature, ${ }^{18}$ also show that PCR-based DNA analysis techniques are also possible on agar pre-embedded tissue, although perhaps re-evaluation should be performed using modern molecular biology techniques.

In conclusion, agar pre-embedding of small skin biopsies is inexpensive, safe and easy to use and has shown a positive impact on orientation without causing problems for ancillary techniques. Importantly, a small loss of time in the cut up room can be made up in terms of gain in time at the embedding station; in an era of quality control (indeed our pathology has an active quality control team ${ }^{19}{ }^{20}$ ), being able to objectively collect this kind of data is fundamental for the improvement and safety of pathology laboratory output.

\section{Take home messages}

The agar pre-embedding technique for small skin biopsies resolves problems in orientation.

- It substantially shortens paraffin embedding times while only slightly lengthening cut up times.

- Immunohistochemistry and in situ hybridization are not affected by agar pre-embedding.

Handling editor Dhirendra Govender.
Acknowledgements We wish to thank Ms Simona Pigozzi and Ms Giorgia Chiossone for technical help as well as all the laboratory staff at the ASL 3, Ospedale Villa Scassi and Ospedale Policlinico San Martino IRCCS histopathology laboratories.

Contributors MR, MP, LV: collected the data. SS: performed the FISH analysis. $M G, A P, L A$ : performed the agar pre-embedding at cut up. RF: critically revised the manuscript. FG, LM: wrote the manuscript and devised the study.

Funding The authors have not declared a specific grant for this research from any funding agency in the public, commercial or not-for-profit sectors.

Competing interests None declared.

Patient consent for publication Not required.

Provenance and peer review Not commissioned; externally peer reviewed.

\section{REFERENCES}

1 Lund $\mathrm{HZ}$, Forrest WW, Harris JR, et al. Preliminary embedding in agar-agar. Its value in the processing of multiple separately identified specimens, and in the orientation of small biopsy specimens. Tech Bull Regist Med Technol 1961;31:562-4.

2 Buzzell GR. Double-embedding techniques for light microscope histology. Stain Technol 1975:50:285-7.

3 Cook RW, Hotchkiss GR. A method for handling small tissue fragments in histopathology. Med Lab Sci 1977;34:43-4.

4 Jones MV, Calabresi PA. Agar-gelatin for embedding tissues prior to paraffin processing. Biotechniques 2007:42:569-70.

5 Ventura L, Bologna M, Ventura T, et al. Agar specimen orientation technique revisited: a simple and effective method in histopathology. Ann Diagn Pathol 2001;5:107-9.

6 Grillo F, Bruzzone M, Pigozzi S, et al. Immunohistochemistry on old archival paraffin blocks: is there an expiry date? J Clin Pathol 2017;70:988-93.

7 Bourgeois $\mathrm{N}$, Braspenninckx E, Wijnen M, et al. The agar-paraffin embedding technique applied to small diagnostic biopsies. Stain Technol 1982;57:251-4.

8 Blewitt ES, Pogmore T, Talbot IC. Double embedding in agar/paraffin wax as an aid to orientation of mucosal biopsies. J Clin Pathol 1982;35:365.

9 Arber DA, Johnson RM, Rainer PA, et al. The bone marrow agar section: a morphologic and immunohistochemical evaluation. Mod Pathol 1993;6:592-8.

10 LoRusso FJ, Font RL. Use of agar in ophthalmic pathology: a technique to improve the handling and diagnosis of temporal artery biopsies, subfoveal membranes, lens capsules, and other ocular tissues. Ophthalmology 1999;106:2106-8.

11 Chiarello SE. Agar embedding of curetted biopsy specimens for histopathologic examination. Am J Dermatopathol 1992;14:174-6.

12 Shaw EB, Johnson JM, Watson CG. Correct orientation of specimens for histologic processing. Am J Dermatopathol 1983;5:165-8.

13 Zozumi M, Nakai M, Ito T, et al. New double embedding technique for specimens of endoscopic submucosal dissection using agarose: comparison with other media. J Clin Pathol 2010;63:904-9.

14 Kerstens HM, Robben JC, Poddighe PJ, et al. AgarCyto: a novel cell-processing method for multiple molecular diagnostic analyses of the uterine cervix.J Histochem Cytochem 2000;48:709-18.

15 De Smet I, Chaerle P, Vanneste $S$, et al. An easy and versatile embedding method for transverse sections. J Microsc 2004:213:76-80.

16 Yan P, Seelentag W, Bachmann A, et al. An agarose matrix facilitates sectioning of tissue microarray blocks. J Histochem Cytochem 2007;55:21-4.

17 Yadav L, Thomas S, Kini U. Improvised double-embedding technique of minute biopsies: a mega boon to histopathology laboratory. Indian J Pathol Microbiol 2015:58:12-16

18 Tsao-Wu GS, Weber CH, Budgeon LR, et al. Agarose-embedded tissue arrays for histologic and genetic analysis. Biotechniques 1998;25:614-8.

19 Bragoni A, Gambella A, Pigozzi S, et al. Quality control in diagnostic immunohistochemistry: integrated on-slide positive controls. Histochem Cell Biol 2017;148:569-73.

20 Gambella A, Porro L, Pigozzi S, et al. Section detachment in immunohistochemistry: causes, troubleshooting, and problem-solving. Histochem Cell Biol 2017;148:95-101. 